\title{
Assessment of Femtosecond Laser Induced Periodic Surface Structures on Polymer Films
}

\author{
Esther Rebollar, ${ }^{\text {a }}$ Javier R. Vázquez de Aldana, ${ }^{\mathrm{b}}$ Ignacio Martín-Fabiani, ${ }^{\mathrm{c}}$ Margarita Hernández, \\ Daniel R. Rueda, ${ }^{c}$ Tiberio A. Ezquerra, ${ }^{c}$ Concepción Domingo, ${ }^{c}$ Pablo Moreno ${ }^{b}$ and Marta Castillejo ${ }^{a}$ \\ ${ }_{5}$ Received (in $\left.X X X, X X X\right)$ Xth $X X X X X X X X X 20 X X$, Accepted Xth XXXXXXXXX 20XX \\ DOI: $10.1039 / b 000000 x$
}

In this work we present the formation of laser induced periodic surface structures (LIPSS) on spin-coated thin films of several model aromatic polymers including poly(ethylene terephthalate), poly(trimethylene terephthalate) and poly carbonate bis-phenol A by irradiation with femtosecond pulses of 795 and $265 \mathrm{~nm}$ 10 at fluences well below the ablation threshold. LIPSS are formed with period lengths similar to the laser wavelength and parallel to the direction of laser polarization vector. Formation of LIPSS upon IR irradiation at $795 \mathrm{~nm}$, a wavelength for which the polymers absorb weakly, contrasts with the absence of LIPSS in this spectral range upon irradiation with nanosecond pulses. Real and reciprocal space characterization of LIPSS obtained by Atomic Force Microscopy (AFM) and Grazing Incidence Small

15 Angle X-ray Scattering (GISAXS) respectively, yields well correlated morphological information. Comparison of experimental and simulated GISAXS patterns suggests that LIPSS can be suitably described considering a quasi-one-dimensional paracrystalline lattice and that irradiation parameters have an influence on the order of such a lattice. Fluorescence measurements, after laser irradiation, provide indirect information about dynamics and structure of the polymer at the molecular level. Our results

20 indicate that the LIPSS are formed by interference of the incident and surface scattered waves. As a result of this process, heating of the polymer surface above its glass transition temperature takes place enabling LIPSS formation.

\section{Introduction}

In recent years nanofabrication with femtosecond laser irradiation ${ }_{25}$ has attracted much interest and has been demonstrated to be a promising method for surface nanostructuring of metals, ${ }^{1}$ semiconductors, $^{2}$ and polymers ${ }^{3}$. In particular, laser-induced morphological changes such as the formation of ripples ${ }^{4}, 5$, bubbles $^{6}$, and conical structures ${ }^{7}$, have been extensively studied.

${ }_{30}$ Laser induced periodic surface structures (LIPSS) have been observed substantially near ablation threshold by many authors since the earlier investigations on laser ablation four decades ago. Ripple formation was first observed in semiconductors irradiated with a Q-switched ruby $\operatorname{laser}^{8}$ and, since then, this kind of 35 structures have been generated on a wide variety of materials ${ }^{4,5,9-}$

11 under different conditions. Despite the extensive studies on LIPSS, only a few references report about LIPSS on polymers upon irradiation in the femtosecond regime. ${ }^{12-16}$ This is surprising, in comparison to the wealth of information on laser 40 processing of polymers with pulsed UV-laser light and pulse lengths of, typically, up to tens of nanoseconds ${ }^{17-20}$ at fluences both below and above the ablation threshold. Additionally, for fs irradiation of polymers, LIPSS have been mostly observed at the bottom of ablation craters.

45 The great variety of experimental work on LIPSS was subsumed into various theoretical approaches ${ }^{21-23}$. In the most common type of surface topography, a periodicity of about the wavelength of the laser radiation is observed. This has been attributed to the interference between the incident and scattered 50 light or excited surface waves ${ }^{22,} 24,25$. Interference between incident linearly polarized light and the surface scattered wave generated during the pulse, leads to a periodic modulation of the absorbed light intensity and, consequently to a modulated surface roughening which enhances the development of the structures via 55 feedback. However, periodic surface structures induced by fs laser irradiation (fs-LIPSS) do not fully comply with previous models on LIPSS formation developed for ns laser irradiation ${ }^{22}$, ${ }^{26}$. The interference model was first suggested by Emmony et al. 25 and was later improved by several other authors ${ }^{21,} 22$. ${ }_{60}$ Particularly, the work of Sipe et al. ${ }^{22}$ represents a first-principle theory which takes into consideration the interaction of an electromagnetic wave with a microscopically rough surface and also includes the possible excitation of surface plasmon polaritons (SPP). Also according to Sipe at al. ${ }^{22}$, efficient surface 65 rippling can be enhanced due to the redistribution of the energy from subsequent laser pulses through a feedback effect. In later publications, Young and co-workers, as well as Clark and Emmony, applied this first-principle theory to explain the formation of LIPSS in semiconductors ${ }^{27-29}$ and metals ${ }^{27,29}$. Some 70 other theoretical attempts have been made to describe fs-LIPSS 
based on well-known models from hydrodynamics of thin films ${ }^{30}$ and on the Sipe-Drude model ${ }^{5,31}$ which considers the laser induced changes in the complex refractive index due to transient generation of quasi-free electrons in the conduction band of the 5 solid. It has also been shown experimentally that the laser polarization plays a very important role to determine the orientation of the ripples ${ }^{9,10}$. Together with LIPSS characterized by a period close to the irradiation wavelength, also called low spatial frequency LIPSS (LSFL), in the case of fs irradiation, a 10 different type of periodic structures, or high spatial frequency LIPSS (HSFL), have been obtained ${ }^{5,}$ 9, 32-34. HSFL have periodicities significantly smaller than the irradiation wavelength and are usually observed for irradiation with ultrashort laser pulses, predominantly for frequencies below band-gap excitation 15 of transparent materials and for repetitive irradiation. The origin of HSFL is still quite controversial and to explain the formation of this kind of structures, different mechanisms have been proposed. These include interference effects along with transient changes in the optical properties during laser irradiation ${ }^{35}$, second 20 harmonic generation ${ }^{32}$, excitation of surface plasmon polaritons ${ }^{36}$, or self-organization effects ${ }^{9}$. Other authors ${ }^{10}$ have proposed a different type of mechanism in which the laser irradiation first originates a highly electrostatically unstable surface. This is followed by emission of high momentum surface ions, which in 25 turn induces an appreciable recoil pressure on the substrate. In this case, ripples would arise from relaxation rather than from wave interference effects.

To determine the temperatures involved in the formation of fsLIPSS, a two temperature model (TTM) has been proposed ${ }^{37,38}$. 30 In this model, electrons and lattice subsystems are described separately by their own temperature distribution. The electronic temperature is estimated assuming that the laser energy is first transferred to the electrons, and then the lattice temperature is determined by assuming energy transfer from the electrons to the 35 lattice. This model has been mainly used to simulate the interaction of short pulses with metals, but has also been applied to other materials such as silicon ${ }^{37}$. However, the strong nonequilibrium conditions associated with ultrashort pulsed laser interaction with solids makes a detailed theoretical analysis 40 difficult. In the case of soft materials, such as polymers, numerical simulations of the evolution of temperature distribution on the surface and within the bulk have also been reported, considering again the generation of free electrons ${ }^{39}$.

In this work we investigate the formation of fs-LIPSS on thin 45 polymer films by irradiation with UV $(265 \mathrm{~nm})$ and IR $(795 \mathrm{~nm})$ pulses. LIPSS of periods close to the laser wavelength, and parallel to the polarization direction, were obtained. The nanostructured surfaces were characterized in real space by Atomic Force Microscopy (AFM), and in reciprocal space by ${ }_{50}$ Grazing Incidence Small Angle X-ray scattering (GISAXS), according to a methodology previously described ${ }^{19}$, 40. As reported, diffraction can be complementary to AFM for LIPSS characterization since it provides inspection of larger areas than those observed by AFM (typically up to a few millimeters in ${ }_{55}$ length). Due to the fact that in GISAXS experiments diffraction is induced by a weak evanescent superficial wave, the use of X-ray synchrotron radiation is especially advantageous ${ }^{41}$. Finally, Raman and fluorescence spectroscopies provided information about chemical and structural modification induced by laser 60 irradiation.

\section{Experimental Section}

Three different polymers, poly (ethylene terephthalate) (PET) (Rhodia S80 from RhodiaSter), poly (trimethylene terephthalate) $(\mathrm{PTT})^{42}$ and polycarbonate bisphenol A (PC) (Lexan ML3021A, ${ }_{65}$ SABIC I-P (Innovative Plastics)) were investigated. Some pertinent physical magnitudes are listed in Table 1. Previous to irradiation, UV-visible absorption spectra of the different polymers casted on quartz substrates were recorded by a UVvisible spectrophotometer (Perkin Elmer U/V Lambda 16) in the 70 range of $190-800 \mathrm{~nm}$. The linear absorption coefficients determined for the two different wavelengths (265 and $795 \mathrm{~nm}$ ) employed in the experiments are reported in Table 1. Polymer thin films were prepared by spin-coating on silicon wafers (100) polished on both surfaces. The wafers were previously cleaned 75 with a piranha solution $\left(\mathrm{H}_{2} \mathrm{SO}_{4}: \mathrm{H}_{2} \mathrm{O}_{2} 3: 1\right)$. PET and PTT were solved in trifluoroacetic acid (Sigma-Aldrich, reagent grade $\geq$ 98\%), and PC in chloroform (Riedel-de Haën 99\%), in a concentration of $20 \mathrm{~g} / \mathrm{L}$ for PET and PTT and of $30 \mathrm{~g} / \mathrm{L}$ in the case of PC. A fixed volume of $0.1 \mathrm{~mL}$ of polymer solution was 80 instantly dropped by a syringe on a rectangular $\left(2 \times 2.5 \mathrm{~cm}^{2}\right)$ silicon substrate placed in the center of a rotating horizontal plate. Samples were rotated for $30 \mathrm{~s}$ at $2380 \mathrm{rpm}$. Thickness of the polymer films, determined by AFM by measuring the depth of a scratch, was ca. $150 \mathrm{~nm}$ and the original spin-coated samples ${ }_{85}$ presented a flat surface, with mean roughness value ${ }^{19}\left(R_{a}\right) \leq 1$ $\mathrm{nm}$.

Laser irradiation was carried out in ambient air with the linearly polarized laser beam of an ultrashort pulsed laser system, which consists of a commercial Ti:Sapphire oscillator (Tsunami, ${ }_{90}$ Spectra Physics) and a regenerative amplifier system (Spitfire, Spectra Physics) based on the chirped pulse amplification technique. The system produces linearly polarized 120 fs pulses at $795 \mathrm{~nm}$ with a repetition rate of $1 \mathrm{kHz}$. The pulse energy can reach a maximum of $1 \mathrm{~mJ}$ and it is finely controlled by a half95 wave plate and a linear polarizer. Neutral density filters were used when further energy reduction was required. The average power of the beam is measured with a thermopile detector (407A, Spectra Physics). The transversal mode is nearly a Gaussian TEM00 with a $10 \mathrm{~mm}$ beam diameter (at $1 / \mathrm{e}^{2}$ ).

100 The second harmonic of the fundamental laser output $(398 \mathrm{~nm}$ central wavelength) was generated in a $1 \mathrm{~mm}$ thick BBO crystal (type-I, $\theta=29^{\circ}$ ), and the third harmonic (265 nm central wavelength) was obtained in a second BBO crystal (1 mm thick, type-I, $\theta=44^{\circ}$ ) by sum-frequency mixing of the fundamental

105 Table 1 Weight average molecular weight $\left(M_{w}\right)$, number average molecular weight $\left(M_{n}\right)$, melting temperature $\left(T_{m}\right)$ and glass transition temperature $\left(T_{g}\right)$ as determined by calorimetry, and linear absorption coefficients $(\alpha)$ at 265 and $795 \mathrm{~nm}$.

\begin{tabular}{rrrrrrr} 
Material & \multicolumn{1}{l}{$\begin{array}{l}\mathrm{M}_{\mathrm{w}} / \\
\mathrm{g} \mathrm{mol}^{-1}\end{array}$} & $\begin{array}{l}\mathrm{M}_{\mathrm{n}} / \\
\mathrm{g} \mathrm{mol}^{-1}\end{array}$ & $\begin{array}{c}\mathrm{T}_{\mathrm{m}} / \\
{ }^{\circ} \mathrm{C}\end{array}$ & $\begin{array}{c}\mathrm{T}_{\mathrm{g}} / \\
{ }^{\circ} \mathrm{C}\end{array}$ & $\begin{array}{l}\alpha_{265} / \\
\mathrm{cm}^{-1}\end{array}$ & $\begin{array}{c}\alpha_{795} / \\
\mathrm{cm}^{-1}\end{array}$ \\
\hline PET & 27600 & 18800 & 252 & 75 & 18000 & 835 \\
PTT & 42000 & 21000 & 229 & 44 & 26000 & 705 \\
PC & 44400 & 23500 & 267 & 151 & 18000 & 365
\end{tabular}


with the second harmonic beams. An estimation of the temporal duration of these UV pulses is $260 \mathrm{fs}$ (taking into account the group-velocity mismatch of the interacting pulses in the crystal), and the maximum available pulse energy is $40 \mu \mathrm{J}$.

5 For irradiation of samples, the fs laser beam impinged perpendicularly onto the target surface which was placed on a motorized $\mathrm{XYZ}$ translation stage, where the $\mathrm{Z}$ axis is perpendicular to the sample surface. In the case of irradiation at $795 \mathrm{~nm}$, the pulses were focused on the surface by a biconvex, 10 achromatic spherical lens with a focal length of $100 \mathrm{~mm}$, and an uncoated fused silica plano-convex spherical lens with a focal length of $100 \mathrm{~mm}$ was used for irradiation at $265 \mathrm{~nm}$. Dependence of the formed structures on the laser fluence $F$ and number of pulses $N$ was studied. The value of $N$ was selected by 15 an electromechanical shutter. Additionally, samples for GISAXS and Raman analysis, which require larger processed areas, were irradiated by a sample scanning process. The scanning speed, and consequently the spatial overlap of successive pulses, was chosen to ensure the delivery of the optimal amount of pulses for LIPSS 20 formation. The total irradiated area was around $4 \times 4 \mathrm{~mm}^{2}$.

Topography of the polymer films was examined via Atomic Force Microscopy (AFM, Nanoscope IIIA Multimode, Veeco) in tapping mode and images were analysed with the software Nanoscope Analysis 1.10. The periods and heights of LIPSS were 25 determined from AFM analysis by averaging the results of three different areas in each sample. Values of the period were derived from Fast Fourier Transform (FFT) analysis (spatial frequency in the direction perpendicular to the LIPSS). Irradiated areas were also analyzed by GISAXS using the facilities of the BW4 30 beamline at HASYLAB (DESY, Hamburg, Germany). The experimental set-up was described elsewhere ${ }^{19,43}$ but a brief description is as follows. An X-ray beam of wavelength $\lambda=$ $0.13808 \mathrm{~nm}$ and cross section size $(\mathrm{H} \mathrm{x} \mathrm{V})$ of $40 \times 20 \mu \mathrm{m}^{2}$ was used in our experiments. Samples are placed horizontally in such 35 a way that the incident X-ray beam was parallel to the direction of the LIPSS and acquisition times of typically $200 \mathrm{~s}$ were used. In order to do this, the rotational angle between the ripples direction and the X-ray beam was varied and optimized to obtain the best pattern in terms of symmetry. An incidence angle $\alpha_{l}=$ ${ }_{40} 0.4^{\circ}$ was chosen to ensure full penetration in the sample. The scattered intensity was recorded by a Mar CCD detector of 2048 x 2048 pixels with a resolution of $79.1 \mu \mathrm{m}$ per pixel, using a distance sample-to-detector of $2.211 \mathrm{~m}$. Lateral correlation between scattering objects (ripples) on the film surface gives 45 scattered intensity maxima appearing out of the meridian ${ }^{44}$. The treatment of the GISAXS images was performed using the software Fit $2 \mathrm{D}^{45}$. GISAXS measurements provided an independent determination of the LIPSS period, to be compared with the value obtained by AFM.

50 Raman spectra were recorded with a micro-Raman spectrometer (Renishaw InVia 0310-02), equipped with a CCD and using a $532 \mathrm{~nm}$ laser as excitation source, operating at a power level of around $5 \mathrm{~mW}$. The spectra were acquired by collecting scattered light in back scattering geometry, using a 50x 55 magnification objective. Acquisition time and accumulations were $10 \mathrm{~s}$ and 10 respectively, and the spectral resolution was 2 .
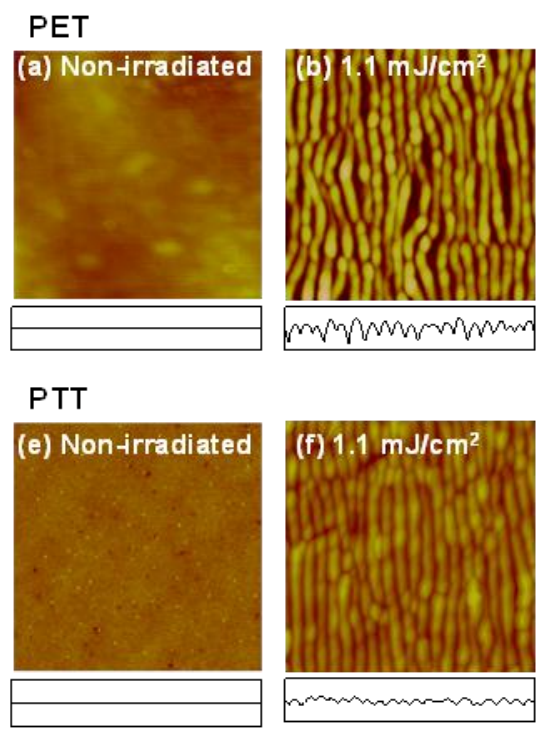

$\mathrm{PC}$
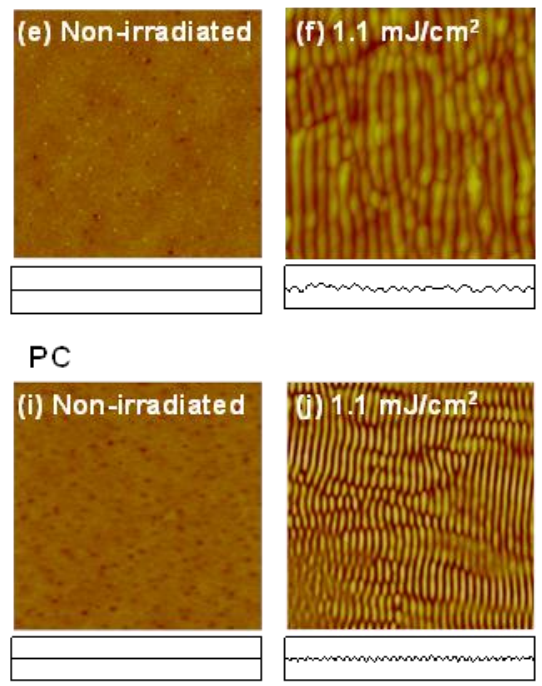
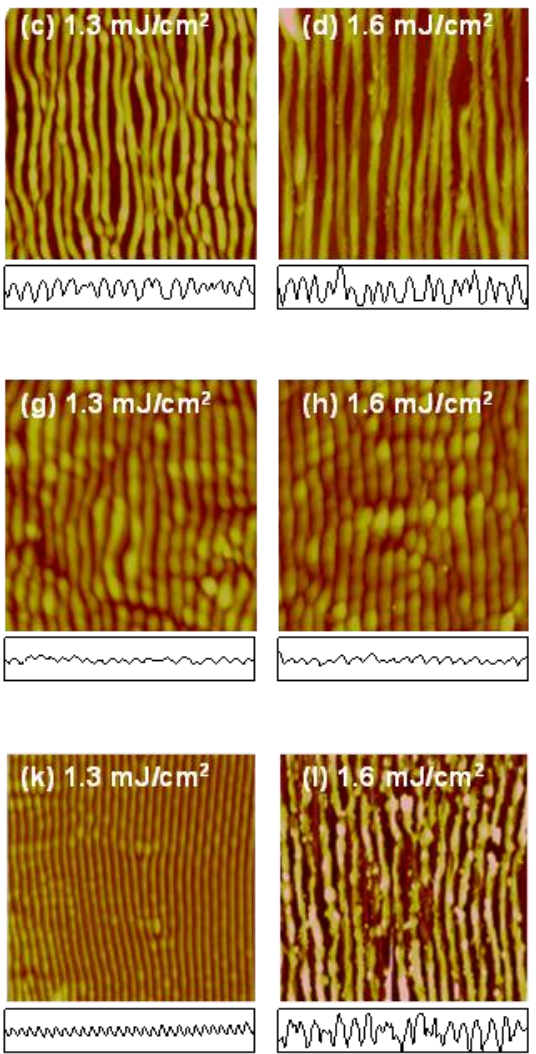

Figure 1 AFM height images $\left(5 \times 5 \mu \mathrm{m}^{2}\right)$ of PET, PTT and PC irradiated at $265 \mathrm{~nm}$ with 5000 pulses at the indicated fluences. Images of as-spun polymers are shown as reference. The height profile along a $5 \mu \mathrm{m}$ line perpendicular to the ripples is shown below every image (height scale: $250 \mathrm{~nm}$ ). 


\section{Cite this: DOI: $10.1039 / \mathrm{c0xx00000x}$}

$\mathrm{cm}^{-1}$. Fluorescence spectra were also acquired with the same instrument and excitation source $(532 \mathrm{~nm})$ using again a $50 \mathrm{x}$ magnification objective. A power of $25 \mathrm{~mW}$ was applied for recording the fluorescence spectra of polymers, both non5 irradiated and irradiated at $795 \mathrm{~nm}$. For samples irradiated at $265 \mathrm{~nm}$, laser excitation powers were limited to $0.5 \mathrm{~mW}$ (PTT) and $0.05 \mathrm{~mW}$ (PET and PC) to avoid sample burning. Additional fluorescence measurements were performed by excitation at 325 $\mathrm{nm}$, a wavelength commonly used for measuring polymer 10 fluorescence. In this case, an objective for NUV of $15 \mathrm{x}$ was employed, while the power for measurements was kept very low, $0.01 \mathrm{~mW}$, in order to avoid damage of the polymer film surface

\section{Results}

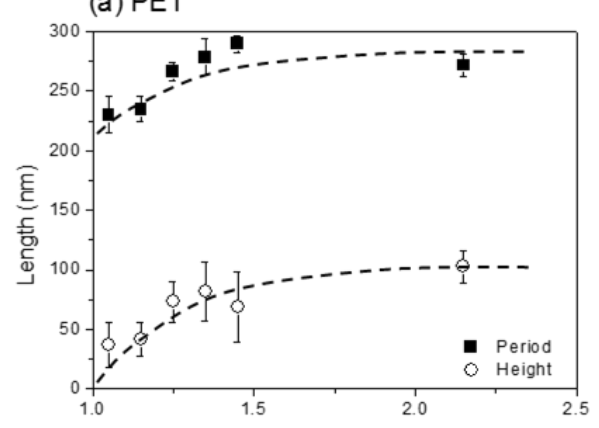

(b) PTT Fluence $\left(\mathrm{mJ} / \mathrm{cm}^{2}\right)$

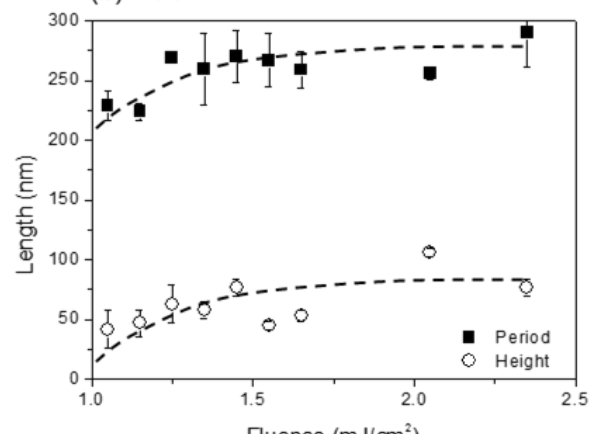

(c) PC

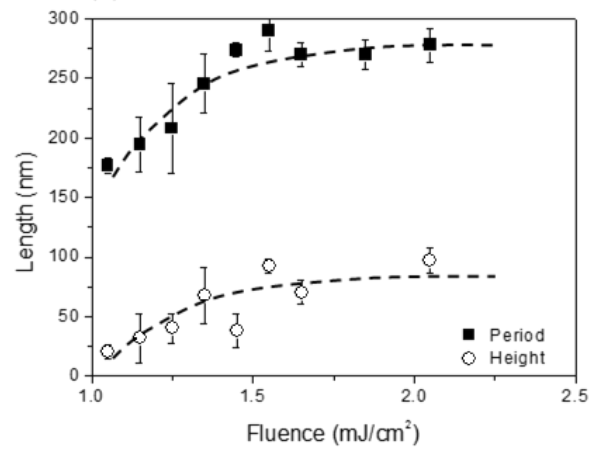

\subsection{AFM}

15 After laser irradiation within an appropriate fluence range, well below the ablation threshold, and with a given number of pulses, ripples aligned parallel to the polarization vector of the laser beam were observed. It is important to point out that these periodic structures were not created at the bottom of ablation 20 craters but on the original film surface. In fact, the AFM analysis of the boundary between a non-irradiated and an irradiated area, shows that the peaks and valleys of the ripples lie respectively above and below the initial surface level ${ }^{15}$. The height of the untreated surface is equivalent to the average height of the 25 nanostructured region of the polymer film, thus confirming that the amount of material removed during laser irradiation is
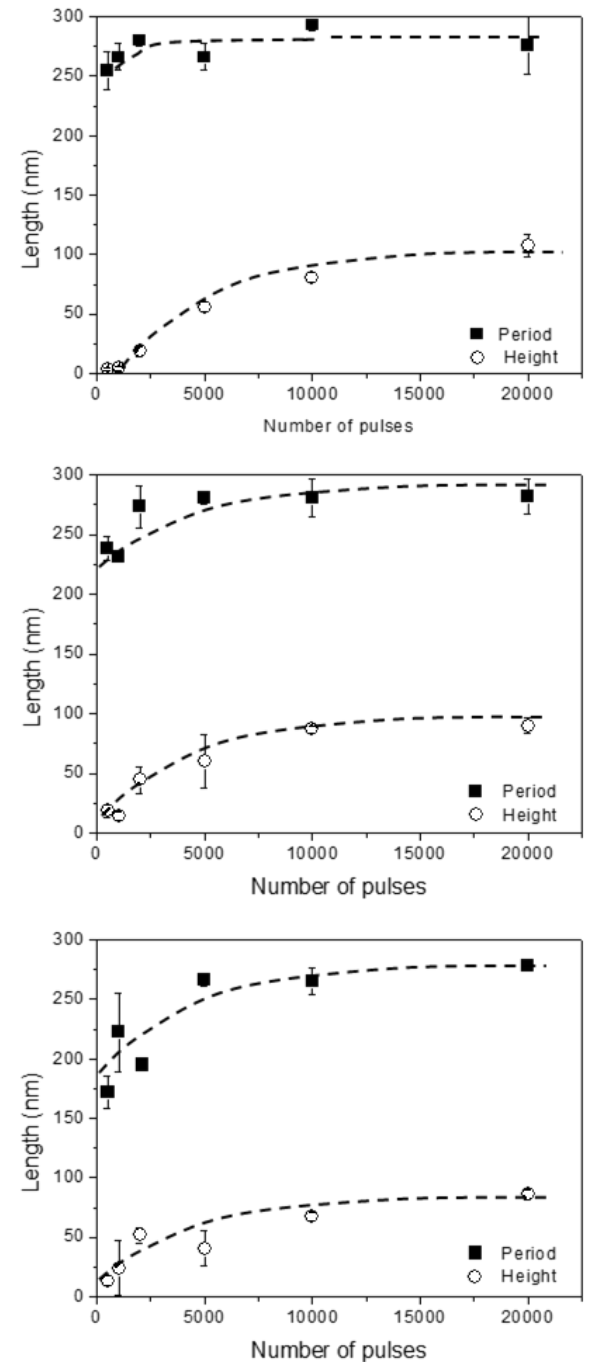

Figure 2 Period and height of LIPSS formed upon irradiation at $265 \mathrm{~nm}$ with 5000 pulses as a function of irradiation fluence (left) and at a fluence of 1.4 $\mathrm{mJ} / \mathrm{cm}^{2}$ as a function of the number of pulses (right) for (a) PET, (b) PTT and (c) PC. The lines are plotted as visual guides. 


\section{Cite this: DOI: 10.1039/c0xx00000x}
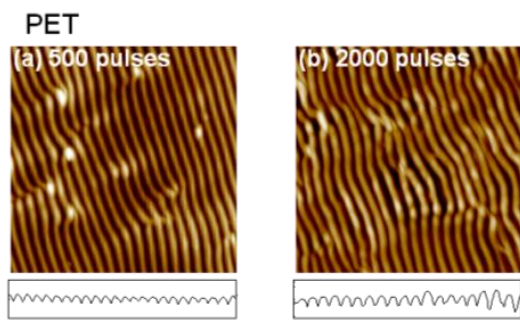

PTT

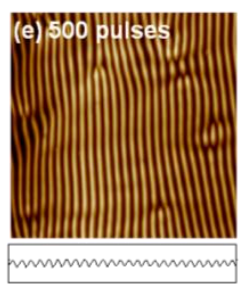

$\mathrm{PC}$

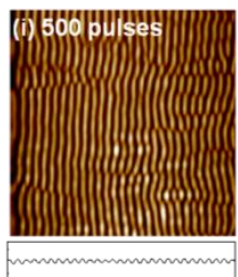

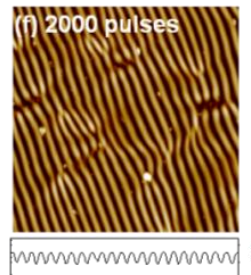

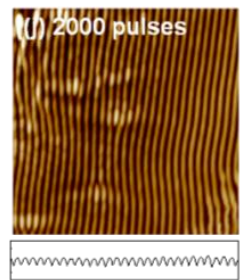

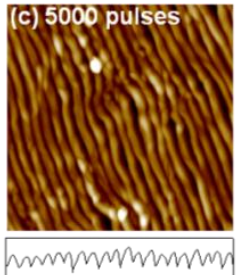
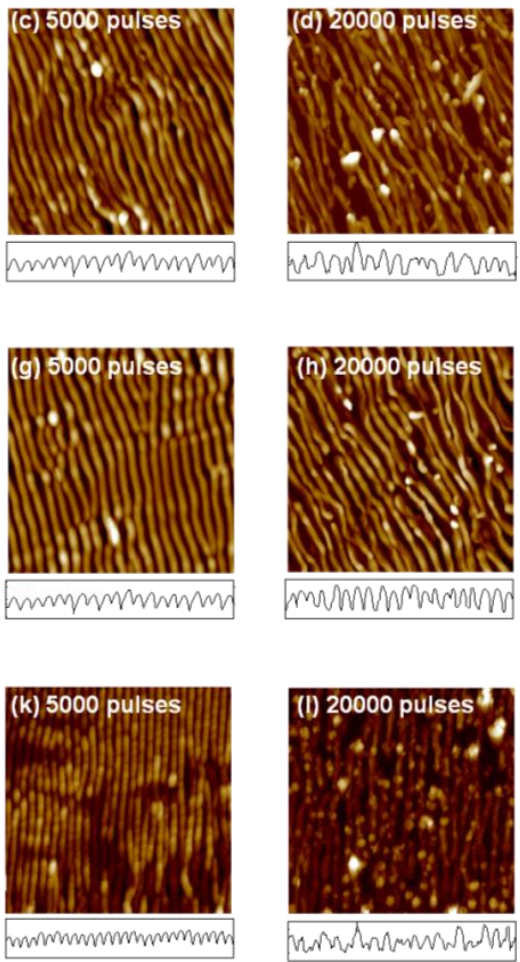

Figure 3 AFM height images $\left(5 \times 5 \mathrm{~m}^{2}\right.$ ) of PET, PTT and PC irradiated at $265 \mathrm{~nm}$ with different number of pulses, as indicated, at the fluence 1.4 $\mathrm{mJ} / \mathrm{cm}^{2}$. The height profile along a $5 \mu \mathrm{m}$ line perpendicular to the ripples is shown below every image (height scale: $250 \mathrm{~nm}$ ).

negligible and that the structures originate from a self5 organization process not involving ablation.

Figure 1 displays typical AFM height images of areas of PET,PTT and PC irradiated at $265 \mathrm{~nm}$ with 5000 pulses at different fluences. For low fluences $\left(F<1 \mathrm{~mJ} / \mathrm{cm}^{2}\right)$, no morphological changes are induced on the polymer surface and 10 the roughness remains constant. At around $1 \mathrm{~mJ} / \mathrm{cm}^{2}$, structures start to be formed and develop the form of ripples, which are parallel and well defined for fluences above $1 \mathrm{~mJ} / \mathrm{cm}^{2}$. At approximately $1.6-1.9 \mathrm{~mJ} / \mathrm{cm}^{2}$ (slightly higher values in the case of PC) LIPSS start to distort. Finally, disruption and ablation of 15 the polymer film takes place at even higher values of $F$, above 2.3 $\mathrm{mJ} / \mathrm{cm}^{2}$. 15

The period and height of the structures are plotted in Figure 2. It is observed that both magnitudes increase with fluence until a plateau is reached at ca. $1.5 \mathrm{~mJ} / \mathrm{cm}^{2}$. The final period is of the 20 order of the laser wavelength. As shown, similar results were obtained for all the three polymers for the same range of fluences. It has to be noticed that irradiation of the spin-coated films with pulses of $6 \mathrm{~ns}$ at $266 \mathrm{~nm}$ also yielded LIPSS with wavelengthsized period ${ }^{19}$.

25 In the same way, the dependence of LIPSS dimensions on the number of pulses for a constant fluence was also evaluated. Particularly, for irradiation at $1.4 \mathrm{~mJ} / \mathrm{cm}^{2}$ the corresponding AFM height images are shown in Figure 3.
Repetitive irradiation was needed for LIPSS formation, 30 confirming that a feedback mechanism must be involved in LIPSS formation, as it is also accepted for irradiation with ns pulses ${ }^{19}$. Again, both the period and the height of the structures increase with $N$ until a plateau is reached after a few thousand pulses, as shown in Figure 2.

35 LIPSS were also observed upon irradiation at $795 \mathrm{~nm}$ in a narrow range of fluences. Figure 4 shows AFM images of PET and PTT irradiated at $795 \mathrm{~nm}$ with 50000 pulses and different fluences.

LIPSS are clearly observed at 35 and $36 \mathrm{~mJ} / \mathrm{cm}^{2}$. However, 40 above $37 \mathrm{~mJ} / \mathrm{cm}^{2}$, the polymer thin film structure appears distorted and higher fluences induce ablation of the film surface. The period and height of ripples as a function of $F$ in this case are shown in Figure 5.

Periods of the ripples are again of the order of the laser 45 wavelength and their height is systematically larger than that observed in the case of UV irradiation. The dependence of ripple parameters with the number of pulses was also studied. The main difference with the results obtained upon UV irradiation concerns the larger number of pulses needed to develop the periodic 50 structures. Figure 6 shows, as an example, AFM height images of LIPSS formed upon irradiation with different number of pulses at constant irradiation fluence of $37 \mathrm{~mJ} / \mathrm{cm}^{2}$, the corresponding period and height being shown in Figure 7. 

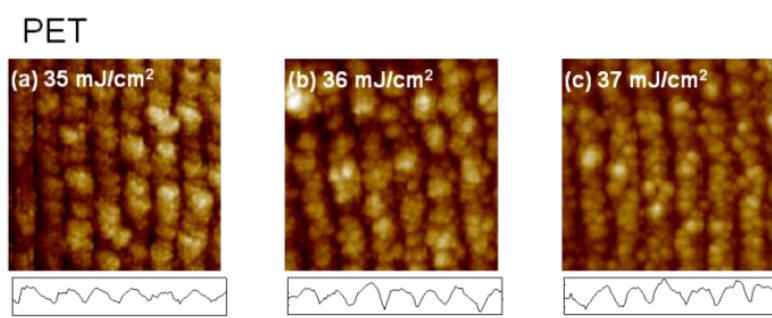

\section{PTT}
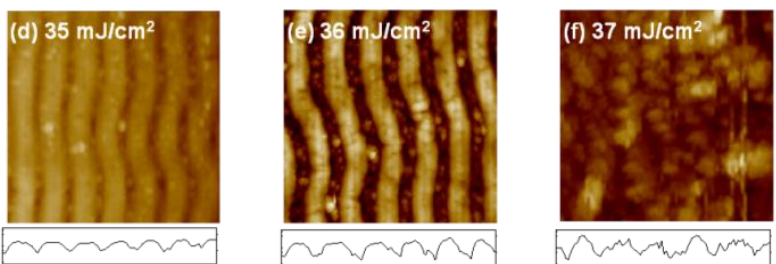

Figure 4 AFM height images $\left(5 \times 5 \mathrm{~m}^{2}\right)$ of PET and PTT irradiated at $795 \mathrm{~nm}$ with 50000 pulses at the indicated fluences. The height profile along a $5 \mu \mathrm{m}$ line perpendicular to the ripples is shown below every image (height scale: $250 \mathrm{~nm}$ ).

\subsection{GISAXS}

GISAXS is a powerful technique, which is gaining more and more interest for the investigation of nanostructures ${ }^{46,47}$. The GISAXS scattering pattern provides structural information over a 10 large sample volume covered by the footprint of the impinging Xray beam on the sample surface of typically up to a few millimeters in the X-ray beam direction, while AFM provides mostly information of a microscopic superficial region.

Figure 8 shows, as an example, characteristic GISAXS 15 patterns of representative rippled polymer films formed by laser irradiation with fs pulses. This type of structures typically presents a scattering pattern consisting of elongated vertical rods along $-\omega$-axis, which are consecutive orders of a first one whose spacing is the reciprocal value of the ripple period ${ }^{48}$. Figure 8 also 20 shows cuts of the scattered intensity along the $\omega$-axis, extracted from the GISAXS patterns at an exit angle $\alpha=0.2^{\circ}$. Spacing of the first lateral maximum from the GISAXS patterns, $L$, can be determined through the expression $L=2 \pi / q_{y}{ }^{\max }$, where $q_{y}{ }^{\max }$ is the reciprocal scattering vector defined ${ }^{46}$ as $q_{y}=$ $25(2 \pi / \lambda) \sin \omega \cos \alpha$. For our irradiated polymers it was found that the spacing values are in well agreement with the periods obtained by AFM. The periods determined by GISAXS from the patterns of Figure 8 are 231, 238 and 245 nm, for PET, PTT and $\mathrm{PC}$ respectively.

30 To gain knowledge about the origin of the observed scattering

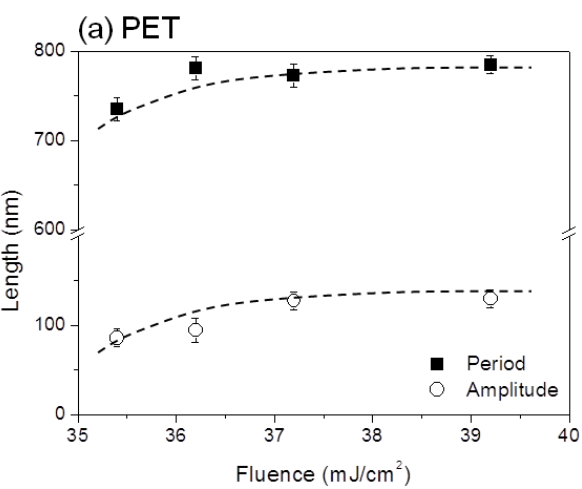

(b) PTT

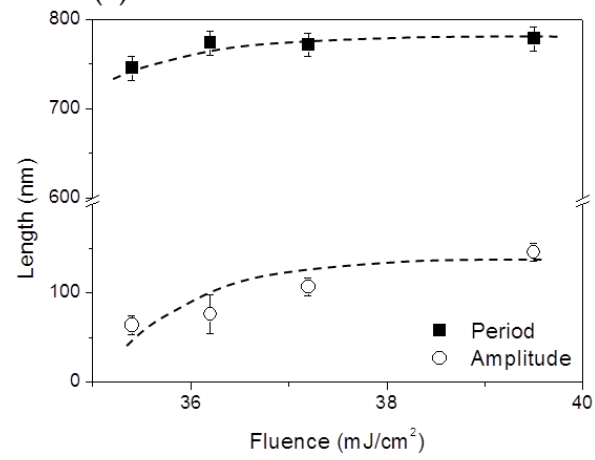

Figure 5 Period and height of LIPSS formed upon irradiation at $795 \mathrm{~nm}$ with 50000 pulses as a function of irradiation fluence for (a) PET and (b) PTT. The lines are plotted as visual guides.

35 features we carried out simple simulations of the GISAXS patterns by using the software package IsGISAXS ${ }^{45,49,50}$. The Distorted Wave Born Approximation (DWBA) was used, assuming a simple nanostructure consisting of polymer boxes standing on the polymer film. As reported previously, ${ }^{40}$ the boxes 40 used to model the nanostructures have typical dimensions as determined by AFM, i.e., width $(2 R)$, height $(H)$, period $(L)$, and length $(W)$. The values used for the simulations shown in Figure 8 are listed in Table 2, and Gaussian distributions for $R$ and $H$ with $\sigma_{R} / R$ and $\sigma_{H} / H$ ca. 0.1 were assumed, being $\sigma_{R}$ and $\sigma_{H}$ the width 45 of the distributions. The parallel boxes were arranged at the surface and a one-dimensional paracrystalline lattice approach was assumed similarly as we did for LIPSS obtained upon irradiation with ns pulses ${ }^{40}$. In a paracrystal the long range order disappears gradually in a probabilistic way ${ }^{45-47,51}$. The probability 50 of finding a particle at a distance $L$ is defined by a function $p(x)$ that was considered as a Gaussian in the present simulation:
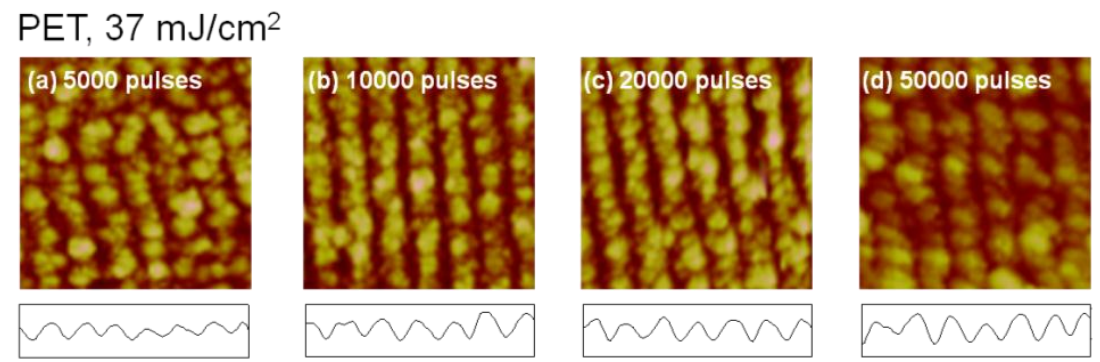

Figure 6 AFM height images $\left(5 \times 5 \mathrm{~m}^{2}\right.$ ) of PET irradiated at $795 \mathrm{~nm}$ with different pulses, as indicated, at the fluence $37 \mathrm{~mJ} / \mathrm{cm}^{2}$. The height profile along a $5 \mu \mathrm{m}$ line perpendicular to the ripples is shown below every image (height scale: $250 \mathrm{~nm}$ ). 


\section{Cite this: DOI: 10.1039/c0xx00000x}

\section{www.rsc.org/xxxxxx}

ARTICLE TYPE

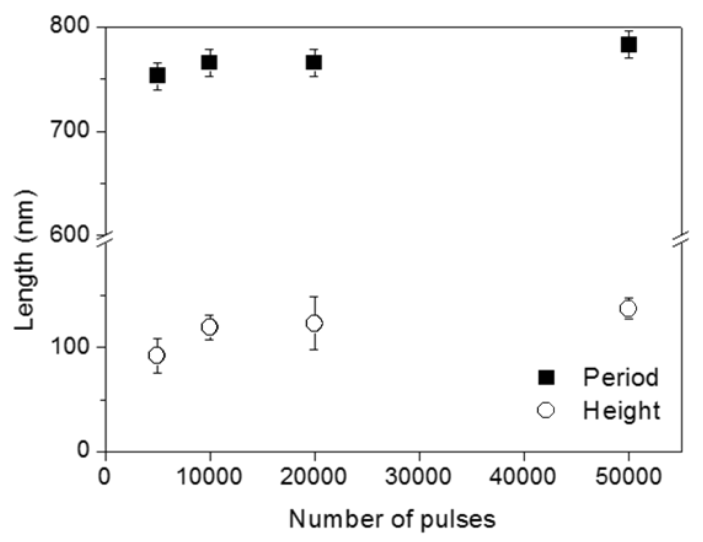

Figure 7 Period and height of LIPSS formed upon irradiation at $795 \mathrm{~nm}$ at a fluence of $37 \mathrm{~mJ} / \mathrm{cm}^{2}$ as a function of the number of pulses for PET. The lines are plotted as visual guides.

${ }_{5} p(x)=\frac{1}{\sigma \sqrt{2 \pi}} \exp \left[-\frac{(x-L)^{2}}{2 \sigma^{2}}\right]$

(Eq. 1)

As the paracrystalline disorder parameter $g=\sigma / L$ increases the lattice disorder increases, while for very small $g$ values, a 1D paracrystal are introduced through the correlation length, $\Delta_{0}^{45}$. Figure 8 (right) shows the simulated GISAXS patterns obtained 10 with the geometrical parameters of the scatterer and the $g$ value considered for the modelling (Table 2). For the sake of comparison, the corresponding intensity profiles at an exit angle of $\alpha=0.2^{\circ}$, normalized to the first correlation peak, both of the experimental and simulated GISAXS patterns, have also been
15 included in Figure 8 (middle panel). These simulations strongly suggest that LIPSS can be quite accurately described considering a quasi-one-dimensional paracrystalline lattice, as it was also reported in the case of LIPSS upon irradiation with ns pulses ${ }^{19,40}$. As the simulation yields information about the order of the 20 structures, through the $g$-parameter, it is possible to derive, for each sample, the optimal irradiation conditions ( $F$ and $N$ ) that yield the lowest value of $g$, and therefore optimize LIPSS formation. Figure 9 shows the dependence of the paracrystalline disorder parameter, $g$, as a function of the laser fluence for 3300 25 pulses. The obtained $g$-values are comparable to those obtained for LIPSS polymer structures using ns pulses ${ }^{40,}{ }^{48}$ It seems that the degree of structural order improves when $F$ increases, and that for this value of $N$ the optimum structural order of LIPPS is achieved at $F \approx 1.5 \mathrm{~mJ} / \mathrm{cm}^{2}$, in agreement with AFM observations. 30 At the highest fluence tested for PC and PTT, a distortion of the structure is evident, probably due to the onset of ablation.

\subsection{Spectroscopic Analysis}

Raman and fluorescence spectra of the nanostructured polymers were recorded in order to obtain information about both the 35 possible chemical modifications and dynamics induced upon laser irradiation. Micro-Raman spectra excited at $532 \mathrm{~nm}$ of the polymers irradiated upon UV light (not shown) display an increase of the fluorescence background with respect to the nonirradiated samples, indicating that additional modifications are 40 induced on the sample surface, besides the topological one. Figure 10 shows the Raman spectra collected on the polymer
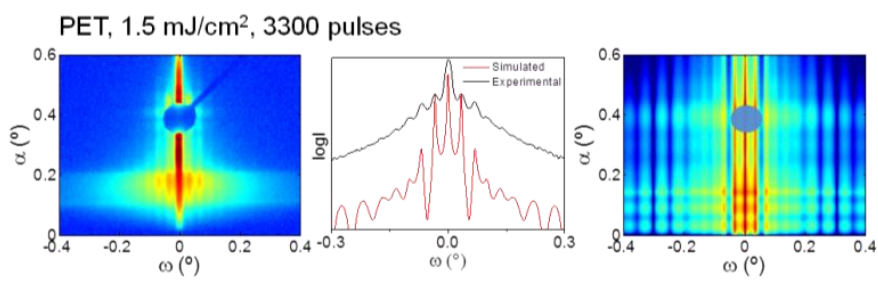

PTT, $1.3 \mathrm{~mJ} / \mathrm{cm}^{2}, 3300$ pulses
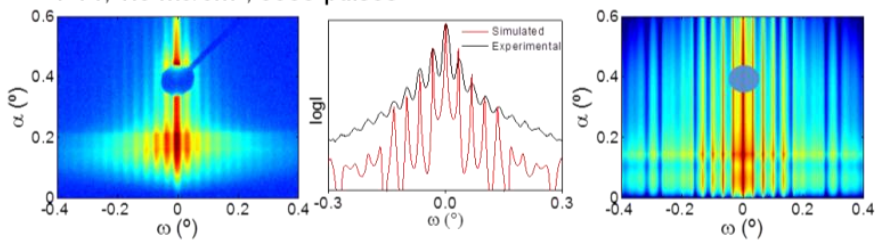

$\mathrm{PC}, 1.5 \mathrm{~mJ} / \mathrm{cm}^{2}, 3300$ pulses
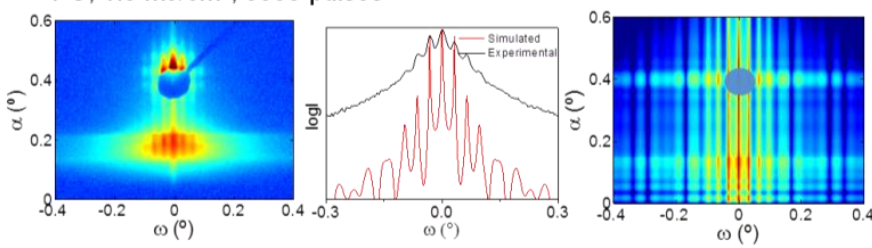

Figure 8 (Left) Experimental GISAXS patterns of PET, PTT and PC irradiated at $265 \mathrm{~nm}$ at the indicated conditions. (Center) Experimental (black lines) and simulated (red lines) intensity profiles taken at a fixed exit angle $\alpha=0.2^{\circ}$. (Right) Corresponding simulated GISAXS patterns. 


\title{
Cite this: DOI: $10.1039 / \mathrm{c0xx00000x}$
}

\author{
www.rsc.org/xxxxxx
}

ARTICLE TYPE

Table 2 Geometric parameters of the scatter (box) used for simulation of GISAXS patterns shown in Figure 8: width $(2 R)$, height $(H)$, length $(W)$, period $(L)$, paracrystalline disorder parameter $(g)$ and correlation length $\left(\Delta_{0}\right)$. Gaussian distributions for $\mathrm{R}$ and $\mathrm{H}$ with $\sigma_{\mathrm{R}} / \mathrm{R}$ and $\sigma_{\mathrm{H}} / \mathrm{H}$ ca. 0.1 were 5 assumed.

\begin{tabular}{ccccccc}
\hline & & & & & & \\
Sample & $2 R / \mathrm{nm}$ & $H / \mathrm{nm}$ & $W / \mathrm{nm}$ & $L / \mathrm{nm}$ & $g$ & $\Delta_{0} / \mathrm{nm}$ \\
\hline PET & 164 & 44 & 1000 & 238 & 0.062 & 2350 \\
PTT & 158 & 68 & 1000 & 234 & 0.060 & 2450 \\
PC & 164 & 100 & 1000 & 246 & 0.069 & 3200
\end{tabular}

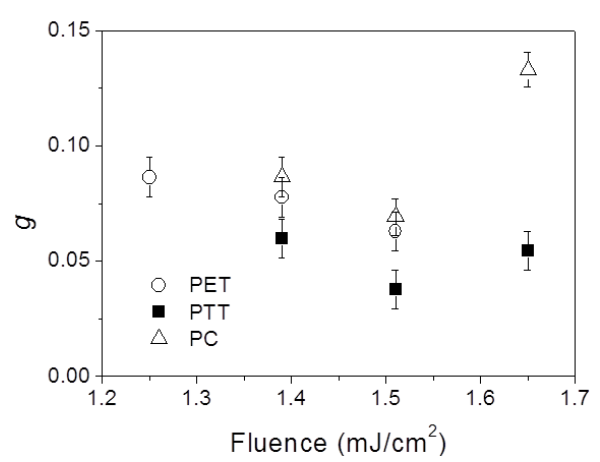

Figure 9 Paracrystalline disorder parameter, $g$, estimated by modelling, as a function of laser fluence for the LIPSS generated by fs laser irradiation at the wavelength of $265 \mathrm{~nm}$, for a number of pulses of 3300 .

samples before and after irradiation at $795 \mathrm{~nm}$, applying 50000 pulses at $37 \mathrm{~mJ} / \mathrm{cm}^{2}$. For PET, the spectra corresponding to the non-irradiated and irradiated films do not differ substantially, while for PTT and PC, the appearance of new weak bands at 15 around 1580 and $1340 \mathrm{~cm}^{-1}$, assigned to amorphous carbon $^{52}$,provide evidence of slight carbonization of the film surface upon irradiation.

Fluorescence spectra of polymer films were recorded upon excitation at the two wavelengths of $532 \mathrm{~nm}$ and $325 \mathrm{~nm}$. Figure

2011 shows the spectra acquired at $532 \mathrm{~nm}$ of samples before and after irradiation. Before irradiation, the spectra of the three polymers present three broad bands with maxima at around 600, 700 and $960 \mathrm{~nm}$, the latter displaying the highest intensity for PET and PTT. LIPSS formation at $265 \mathrm{~nm}$ gives rise to 25 significant changes in the fluorescence spectra. It is observed that the intensity of the bands at 600 and $700 \mathrm{~nm}$ increases, which is related to the increase of fluorescence recorded by exciting the Raman spectra at $532 \mathrm{~nm}$. It is also observed that the relative intensity of these two bands reverses with respect to the non30 irradiated case. Additionally, the band at $960 \mathrm{~nm}$ disappears. Fluorescence of the films nanostructured at $795 \mathrm{~nm}$ show variations of the intensity of the $960 \mathrm{~nm}$ band, which are more pronounced in the case of PC. No information is found in the literature concerning fluorescence emission at the long excitation

35 wavelength of $532 \mathrm{~nm}$, therefore the assignment of the observed bands is uncertain.

More information on fluorescence of the samples was gathered
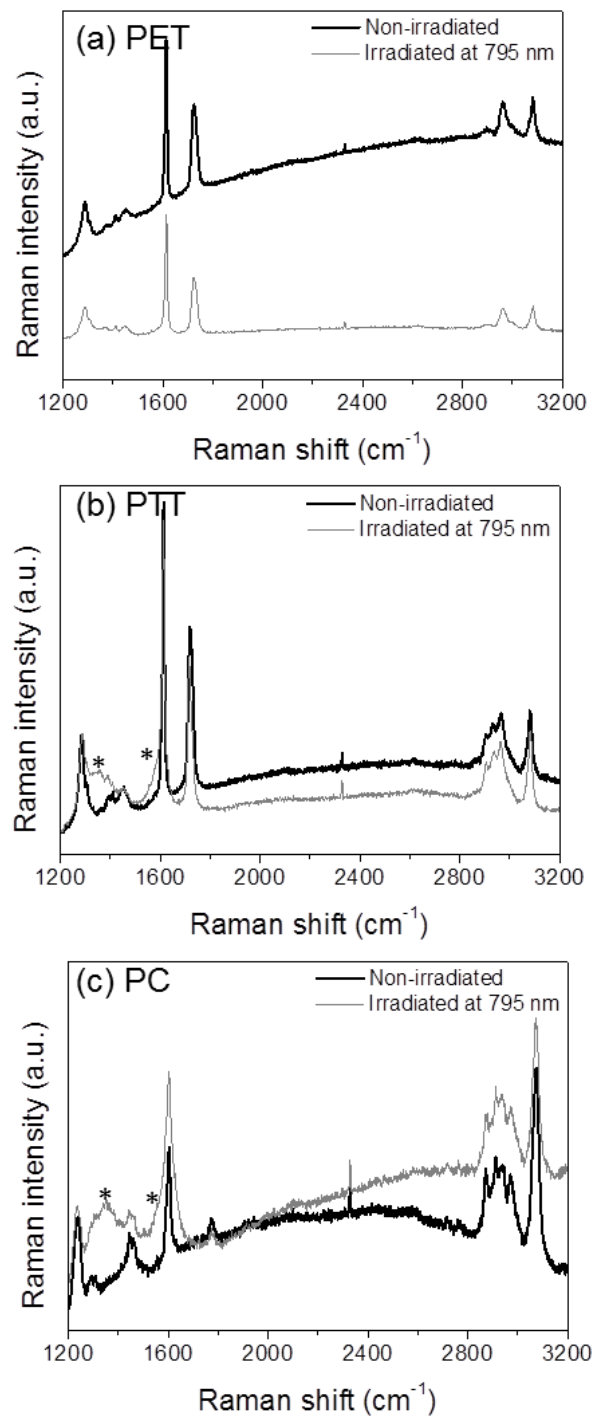

Figure 10 Micro-Raman spectra $\left(\lambda_{\text {laser }}=532 \mathrm{~nm}\right)$ of spin-coated polymer 40 films before (black) and after (grey) irradiation at $795 \mathrm{~nm}$ with 50000 pulses at $37 \mathrm{~mJ} / \mathrm{cm}^{2}$ (a) PET, (b) PTT and (c) PC. Bands assigned to amorphous carbon are indicated with an asterisk.

by recording the spectra at $325 \mathrm{~nm}$, as fluorescence of polymers is typically studied by exciting in the UV region. In this case (see ${ }_{45}$ Figure 12), the spectra of PET and PTT contain two bands at around 390 and $460 \mathrm{~nm}$. These bands are assigned ${ }^{53-55}$ to the main-chain phenylene groups $(390 \mathrm{~nm})$ and to "excimer" or "dimer" formed $(460 \mathrm{~nm})$ as a consequence of the interaction between chromophores, i.e. between polymer chains. LIPSS 50 results in the modification of the relative intensity of these two bands, the one at $390 \mathrm{~nm}$ decreasing in comparison with that at $460 \mathrm{~nm}$, which is related to a different dimerization degree of the phenyl moiety of the polymeric chains.

\section{Discussion}



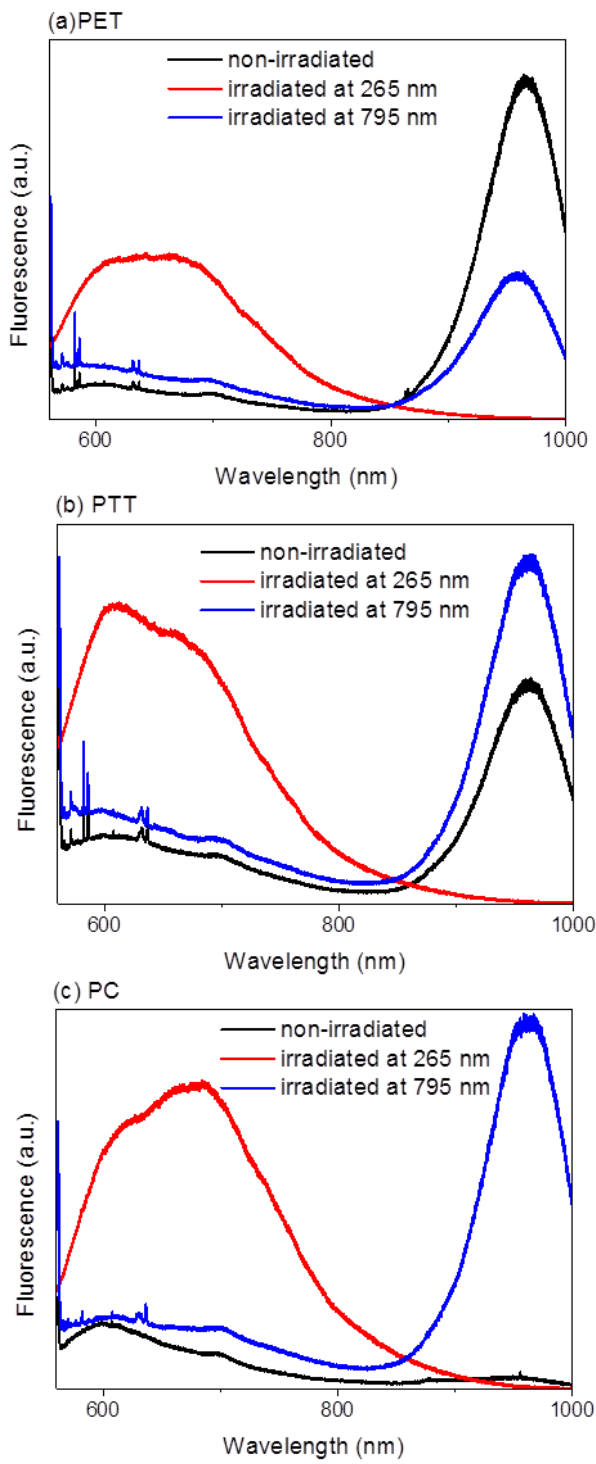

Figure 11 Fluorescence spectra upon excitation at $532 \mathrm{~nm}$ of the spincoated films non-irradiated and irradiated at $265 \mathrm{~nm}$ with 3300 pulses at $1.51 \mathrm{~mJ} / \mathrm{cm}^{2}$, and $795 \mathrm{~nm}$ with 50000 pulses at $37 \mathrm{~mJ} / \mathrm{cm}^{2}$ (a) PET, (b) PTT and (c) PC.

The results presented herein demonstrate LIPSS formation in the non-ablative regime on thin polymer films upon irradiation with fs laser pulses at UV $(265 \mathrm{~nm})$ and IR wavelengths $(795 \mathrm{~nm})$. Although in both cases the periodicity of the ripples nearly 10 coincides with the laser irradiation wavelength, the fluences needed for LIPSS formation using the IR wavelength are much higher. Additional important features of the obtained LIPPS are their alignment parallel to the laser polarization direction, alike to the results shown by Kautek et al. ${ }^{12,13,16}$ for polyimide, and the 15 fact that no ablation is produced by laser processing under the present experimental conditions. These characteristics configure a different scenario as compared with LIPSS obtained in polymer thin films with ns pulses ${ }^{19}, 40$, where the structures are only generated at strongly absorbed wavelengths, and with LIPSS 20 generated on different materials (semiconductors, metals, and dielectrics) ${ }^{4}, 10,14,31,32,37,56$, which show both LSFL and HSFL structures at the bottom of ablation craters.


Figure 12 Fluorescence spectra upon excitation at $325 \mathrm{~nm}$ of the spin25 coated films non-irradiated and irradiated at $265 \mathrm{~nm}$ with 3300 pulses at $1.51 \mathrm{~mJ} / \mathrm{cm}^{2}$, and at $795 \mathrm{~nm}$ with 50000 pulses at $37 \mathrm{~mJ} / \mathrm{cm}^{2}$ (a) PET and (b) PTT.

As the periodicities of the ripples reported here nearly coincide with the laser irradiation wavelengths, the phenomenon can be 30 attributed to scattering, diffraction and subsequent local-field enhancement effects ${ }^{21,22,27-29,32}$. The absence of ablation in the nanostructuring of the thin polymer films upon irradiation at the present conditions, account for a self-organization mediated process which does not require surface material removal. 35 Additionally, a feedback process is clear, as repetitive irradiation is needed in order to observe LIPSS formation. The parallel orientation is supported by the Sipe theory ${ }^{22}$. The orientation of the ripples with respect to the polarization of the incident laser light, depends on the value of the dielectric permittivity of the 40 substrate, $\varepsilon=\varepsilon^{\prime}-1 \varepsilon^{\prime \prime}$, in such a way that the orientation of the ripples is parallel to the polarization when $|\varepsilon|<1$ as is the case of dielectrics $^{57}$ and, in particular, of polymers.

As indicated, LIPSS are obtained upon irradiation at the weakly absorbed (in the linear regime) wavelength of $795 \mathrm{~nm}$. ${ }_{45}$ This is in contrast to experimental observations in the ns case, where no LIPSS are detected in this spectral region. Due to the high intensities involved in the fs regime, non-linear absorption and ionization processes mediate the coupling of laser light with the outer layer of the films, providing the necessary process for ${ }_{50}$ effective absorption. In our case, the relative contribution of multiphoton (MP) and tunnel ionization processes on surface modification can be assessed by calculating the adiabaticity parameter, $\gamma$, introduced by Keldysh ${ }^{58}, \gamma=\left(E_{i} / 1.87 \cdot 10^{-13} I \lambda^{2}\right)^{1 / 2}$, where $E_{i}$ is the molecular ionization potential (in $\mathrm{eV}$ ), $I$ the peak ${ }_{55}$ laser intensity (in $\mathrm{W} \mathrm{cm}^{-2}$ ) and $\lambda$ is given in $\mu \mathrm{m}$. Values obtained 
for both 795 and $265 \mathrm{~nm}$ at the fluences used for LIPSS formation $^{15}$ are well above the unity, indicative of a MP ionization mechanism governing the initial light absorption step. The generation of quasi-free electrons at the early stages of the 5 interaction with the laser pulse has been reported in the case of polymers $^{39}$. As mentioned, in this case only LSFL are observed. The formation of HSFL has been reported in polymers upon irradiation with higher fluences, in the ablation regime ${ }^{34}$. In such a case different mechanisms must be involved, including changes 10 in optical properties ${ }^{35}$, second harmonic generation ${ }^{32}$ and excitation of surface plasmon polaritons ${ }^{36}$.

Upon irradiation with nanosecond laser pulses a very rapid heating of the target material followed by a fast cooling down of the affected volume to the surrounding temperature take place, 15 but despite the short times involved, all these processes take place under conditions of local thermal equilibrium. Differently, upon irradiation in the fs regime, pulses are short enough to address only the target electronic system, while all other effects such as energy transfer to the lattice, ablation, and relaxation, only ${ }_{20}$ develop after the laser pulse has ceased. In the framework of a TTM, as described in the Introduction, the deposition of laser energy into the medium is mediated by the generation of quasifree electrons which, by energy transfer to the substrate, are responsible for the temperature rise. The increase of temperature 25 is approximated by the expression ${ }^{6,39} \Delta T=\varepsilon /\left(\rho_{0} C_{p}\right)$, where $\varepsilon$ is the plasma energy density at the end of the laser pulse and $\rho_{0}$ and $C_{p}$ are respectively the density and the heat capacity of the material. The plasma energy density is given by $\varepsilon=\rho_{c r}$ (9/4) $E_{i}$, $\rho_{c r}$ being the critical free electron density to generate both a 30 strongly reflective and absorbing plasma. As discussed earlier ${ }^{15}$, $\rho_{c r} \alpha(2 \pi / \lambda)^{2} m_{c} e_{0} / e^{2}$, with $\varepsilon_{0}$ the vacuum dielectric permittivity, $m_{c}$ the mass of the quasi-free electron in the conduction band and $e$ the electron charge. Consequently, and given the dependence on the irradiation wavelength, for a given polymer, $\Delta T$ should be 35 higher in the case of irradiation at $265 \mathrm{~nm}$, thus also explaining the larger $N$ and $F$ values needed for LIPSS formation upon fs irradiation at $795 \mathrm{~nm}$.

Related to the temperature increment are the experimental observations of the increase of the ripple period and height as a 40 function of the fluence. Higher fluence leads to an increase of the superficial temperature, which in turn generates a softer material with lower superficial viscosity ${ }^{59}$. This allows the formation of wider structures characterized by larger periods at higher fluences.

45 This fact is also related to the dependence of the degree of structural order of LIPSS; for an optimal number of pulses applied to the sample, the increase of fluence leads to the formation of more ordered structures characterized by a higher degree of structural order and by larger periods, which is 50 demonstrated by the lower value of $g$ obtained from the simulation for the GISAXS patterns. Further irradiation provokes the loss of structural order; at high fluences, the temperature attained on the surface increases, the depth affected increases, and finally the whole film, and not only a superficial layer, is 55 affected. In this case the complete film undergoes devitrification. Under these circumstances film dewetting is likely to occur in addition to ablation of the film material takes place. Similar explanation can be given for the increase of period for larger number of pulses; due to "incubation" effects together to the ${ }_{60}$ feedback mechanism, an increase of superficial temperature occurs, allowing polymer dynamics and LIPSS formation.

Considering the ensuing thermal effects induced by laser irradiation, as previously shown for polymer films irradiated with ns pulses ${ }^{19,} 4^{40}$, the temperature increase needed for LIPSS 65 formation should be above the glass transition temperature and below the melting point of the material. In order to obtain LIPSS, a minimum fluence value is necessary to ensure surface heating above $T_{g}$ to induce surface devitrification and to allow significant polymer segmental and chain dynamics. Temperature increase 70 above $T_{g}$ is expected to raise surface roughness caused by capillary waves, consequently enhancing surface inhomogeneties and facilitating the feedback mechanism involved in LIPSS formation.

Indirect information on dynamics of ripple formation can be 75 obtained from the fluorescence spectra. As mentioned, the fluorescence band at $390 \mathrm{~nm}$ observed upon UV excitation, has its origin in the aromatic polymer moiety that contains the terephthalic units. Since the ring is incorporated into the chain backbone, fluorescence from the ring is capable to provide 80 accurate information about the rearrangement of phenyl moieties of the polymer at the molecular level. The relative intensity increase of the longer wavelength band observed at $460 \mathrm{~nm}$, and assigned to excimer fluorescence, suggests a larger interaction among chromophores and/or higher level of dimers on the 85 nanostructured surfaces. This is likely a consequence of the rearrangement of polymer chains after irradiation and subsequent heating above the glass transition temperature.

Spectroscopic analysis gives also information on chemical changes. Fluorescence increase caused by nanostructuring with ${ }_{90} \mathrm{UV}$ laser light could be ascribed to the formation of additional fluorescent species, e.g. carbonyl groups and double bonds. In fact, oxidation and slight chemical modifications of polymer surfaces during LIPSS formation have been previously reported for irradiation with UV pulses in the ns regime ${ }^{18,40}$ while upon 95 irradiation with IR laser light at $795 \mathrm{~nm}$ Raman spectroscopy shows a slight carbonization on PTT and PC, and a negligible effect on PET. Further studies are in progress in order to assign the emission band observed at $960 \mathrm{~nm}$ upon excitation at $532 \mathrm{~nm}$, an effect which seems to be also related to nanostructuring. ${ }_{100}$ Fluorescence in the NIR region can be important for applications.

\section{Conclusions}

LIPSS parallel to the laser polarization vector are formed on the surface of spin-coated thin films of the polymers PET, PTT and PC upon irradiation with femtosecond pulses both at 795 and 265 $105 \mathrm{~nm}$. The period of the structures is similar to the laser irradiation wavelength. Formation of LIPSS upon IR irradiation at $795 \mathrm{~nm}$, a wavelength for which the polymers absorb weakly in the linear regime, contrasts with the absence of LIPSS in this spectral range upon irradiation with nanosecond pulses. We thus demonstrate 110 the possibility of tuning and controlling the morphology and size of periodic structures on the surface of thin polymer films by operating in the fs regime and by changing the irradiation wavelength, the fluence and the number of pulses. LIPSS formation in the femtosecond regime results from multiphoton 115 absorption and ionization taking place at the early stages of the 
laser material interaction. Heating of the polymer surface at temperatures above the glass transition allows the softening of the material and decrease of viscosity facilitating enough mobility and enabling the formation of paracrystalline gratings.

\section{${ }_{5}$ Acknowledgements}

Funding from MICINN, Spain, (Projects CTQ2010-15680, FIS2009-09522, MAT2012-33517 and CSD2007-00013) and Junta de Castilla y León (Project SA086A12-2) is gratefully acknowledged. The experiments performed at BW4 in

10 HASYLAB (DESY, Germany) were supported by the European Union (Contract RII3-CT-2004-506008 (IA-SFS)). We thank J. Perlich for his support during the measurements at HASYLAB. E.R., I.M.-F and M.H. thank MINECO (previous MICINN), Spain, for a Ramón y Cajal contract (RYC-2011-08069), a FPI 15 fellowship and a PTA contract, respectively. We thank Z. Roslaniec and A. Szymczyk for providing the PTT samples.

\section{Notes and references}

${ }^{a}$ Instituto de Química Física Rocasolano, IQFR-CSIC, Serrano 119, 28006 Madrid, Spain.E-mail: e.rebollar@iqfr.csic.es

$2{ }^{b}$ Grupo de Investigación en Microprocesado de Materiales con Láser, Universidad de Salamanca, Plaza de la Merced s/n, 37008 Salamanca, Spain

${ }^{c}$ Instituto de Estructura de la Materia, IEM-CSIC, Serrano 119-121, 28006 Madrid, Spain

25

1. A. Pereira, A. Cros, P. Delaporte, S. Georgiou, A. Manousaki, W. Marine and M. Sentis, Appl. Phys. A, 2004, 79, 1433-1437.

2. R. A. Ganeev and T. Q. Jia, Optics and Spectroscopy (English translation of Optika i Spektroskopiya), 2008, 105, 141-146.

30 3. E. Rebollar, D. Cordero, A. Martins, S. Chiussi, R. L. Reis, N. M. Neves and B. León, Appl. Surf. Sci., 2011, 257, 4091-4095.

4. J. Reif, O. Varlamova, M. Ratzke, M. Schade, H. Leipner and T. Arguirov, Appl. Phys. A, 2010, 101, 361-365.

5. J. Bonse and J. Kruger, J. Appl. Phys., 2010, 108, 034903-034905.

35 6. S. Gaspard, M. Oujja, R. de Nalda, C. Abrusci, F. Catalina, L. Bañares, S. Lazare and M. Castillejo, Appl. Surf. Sci., 2007, 254, 1179-1184.

7. J. Bonse, S. Baudach, J. Krüger, W. Kautek and M. Lenzner, Appl. Phys. A, 2002, 74, 19-25.

40 8. M. Birnbaum, J. Appl. Phys., 1965, 36, 3688-3689.

9. J. Reif, F. Costache, M. Henyk and S. V. Pandelov, Appl. Surf. Sci., 2002, 197-198, 891-895.

10. F. Costache, M. Henyk and J. Reif, Appl. Surf. Sci., 2002, 186, 352357.

45 11. A. Y. Vorobyev and C. Guo, Appl. Phys. A, 2007, 86, 321-324.

12. S. Baudach, J. Bonse and W. Kautek, Applied Physics A: Materials Science and Processing, 1999, 69, S395-S398.

13. M. Forster, W. Kautek, N. Faure, E. Audouard and R. Stoian, Phys. Chem. Chem. Phys., 2011, 13.

50 14. J. Krüger and W. Kautek, in Polymers and Light, ed. T. K. Lippert, Springer Berlin / Heidelberg, 2004, vol. 168, pp. 247-290.

15. E. Rebollar, J. R. V. de Aldana, J. A. Perez-Hernandez, T. A Ezquerra, P. Moreno and M. Castillejo, Appl. Phys. Lett., 2012, 100, 041106-041104.

55 16. S. Baudach, J. Krüger and W. Kautek, Rev. Laser Eng. , 2001, 29, 705-709.

17. M. Bolle, S. Lazare, M. Le Blanc and A. Wilmes, Appl. Phys. Lett., 1992, 60, 674-676.

18. E. Rebollar, I. Frischauf, M. Olbrich, T. Peterbauer, S. Hering, J.

60 Preiner, P. Hinterdorfer, C. Romanin and J. Heitz, Biomaterials, 2008, 29, 1796-1806.
19. E. Rebollar, S. Pérez, J. J. Hernández, I. Martín-Fabiani, D. R. Rueda, T. A. Ezquerra and M. Castillejo, Langmuir, 2011, 27, 55965606.

65 20. P. E. Dyer and R. J. Farley, Appl. Phys. Lett., 1990, 57, 765-767.

21. Z. Guosheng, P. M. Fauchet and A. E. Siegman, Phys. Rev. B, 1982, 26, 5366-5381.

22. J. E. Sipe, J. F. Young, J. S. Preston and H. M. van Driel, Phys. Rev. $B, 1983,27,1141$.

70 23. A. Barborica, I. N. Mihailescu and V. S. Teodorescu, Phys. Rev. B, 1994, 49, 8385-8395.

24. D. Bäuerle, Laser processing and chemistry, Springer-Verlag, Berlin, 2000.

25. D. C. Emmony, R. P. Howson and L. J. Willis, Appl. Phys. Lett., 1973, 23, 598-600.

26. H. M. van Driel, J. E. Sipe and J. F. Young, Phys. Rev. Lett., 1982, 49, 1955-1958.

27. J. F. Young, J. S. Preston, H. M. van Driel and J. E. Sipe, Phys. Rev. $B, 1983,27,1155$

80 28. J. F. Young, J. E. Sipe and H. M. van Driel, Phys. Rev. B, 1984, 30 , 2001.

29. S. E. Clark and D. C. Emmony, Phys. Rev. B, 1989, 40, 2031-2041.

30. M. Bestehorn, A. Pototsky and U. Thiele, The European Physical Journal B - Condensed Matter and Complex Systems, 2003, 33, 457$85 \quad 467$

31. D. Dufft, A. Rosenfeld, S. K. Das, R. Grunwald and J. Bonse, $J$. Appl. Phys., 2009, 105, 034908-034909.

32. J. Bonse, M. Munz and H. Sturm, J. Appl. Phys., 2005, 97, 013538013539.

90 33. J. Heitz, E. Arenholz, D. Bäuerle, R. Sauerbrey and H. M. Phillips, Applied Physics A, 1994, 59, 289-293.

34. J. M. Guay, A. Villafranca, F. Baset, K. Popov, L. Ramunno and V. R. Bhardwaj, New Journal of Physics, 2012, 14, 085010.

35. Q. Wu, Y. Ma, R. Fang, Y. Liao, Q. Yu, X. Chen and K. Wang, Appl. Phys. Lett., 2003, 82, 1703-1705.

36. G. Miyaji and K. Miyazaki, Opt. Express, 2008, 16, 16265-16271.

37. T. J. Derrien, T. Sarnet, M. Sentis and T. E. Itina, Journal of Optoelectronics and Advanced Materials, 2010, 12, 610-615.

38. S. I. Anisimov, B. L. Kapeliovich and T. L. Perel'man, Sov. Phys. JETP, 1974, 39, 375.

39. A. Vogel, J. Noack, G. Hüttman and G. Paltauf, Applied Physics B. Lasers and Optics, 2005, 81, 1015-1047.

40. I. Martín-Fabiani, E. Rebollar, S. Pérez, D. R. Rueda, M. C. GarcíaGutiérrez, A. Szymczyk, Z. Roslaniec, M. Castillejo and T. A. Ezquerra, Langmuir, 2012, 28, 7938-7945.

41. P. Müller-Buschbaum, Analytical and Bioanalytical Chemistry, 2003, 376, 3-10.

42. S. Anna, European Polymer Journal, 2009, 45, 2653-2664.

43. S. V. Roth, R. Dohrmann, M. Dommach, M. Kuhlmann, I. Kroger, R. Gehrke, H. Walter, C. Schroer, B. Lengeler and P. MullerBuschbaum, Review of Scientific Instruments, 2006, 77, 085106085107.

44. D. R. Rueda, A. Nogales, J. J. Hernández, M.-C. García-Gutiérrez, T. A. Ezquerra, S. V. Roth, M. G. Zolotukhin and R. Serna, Langmuir, 2007, 23, 12677-12681.

45. R. Lazzari, The FIT2D Home Page. http://www.esrf.eu/computing/scientific/FIT2D/, accessed March 15, 2010

46. P. Müller-Buschbaum, in Applications of Synchrotron Light to $120 \quad$ Scattering and Diffraction in Materials and Life Sciences. , eds. T. A. Ezquerra, M. C. García-Gutiérrez, A. Nogales and M. A. Gómez, Springer-Verlag, Berlin, 2009, vol. 776, pp. 61-89.

47. G. Renaud, R. Lazzari and F. Leroy, Surface Science Reports, 2009 , 64, 255-380.

125 48. D. R. Rueda, I. Martin-Fabiani, M. Soccio, N. Alayo, F. PerezMurano, E. Rebollar, M. C. Garcia-Gutierrez, M. Castillejo and T. A. Ezquerra, Journal of Applied Crystallography, 2012, 45, 1038-1045.

49. R. Meier, H.-Y. Chiang, M. A. Ruderer, S. Guo, V. Körstgens, J. Perlich and P. Müller-Buschbaum, Journal of Polymer Science Part B: Polymer Physics, 2012, 50, 631-641.

50. R. Lazzari, Journal of Applied Crystallography, 2002, 35, 406-421. 
51. A. M. Hindeleh and R. Hosemann, J. Mater. Sci., 1991, 26, 51275133.

52. R. O. Dillon, J. A. Woollam and V. Katkanant, Phys. Rev. B, 1984, 29, 3482-3489.

5 53. N. S. Allen and J. F. McKellar, Die Makromolekulare Chemie, 1978, 179, 523-526.

54. M. F. Sonnenschein and C. M. Roland, Polymer, 1990, 31, $2023-$ 2026.

55. W.-a. Luo, Z. Liao, J. Yan, Y. Li, X. Chen, K. Mai and M. Zhang, 10 Macromolecules, 2008, 41, 7513-7518.

56. J. Bonse, A. Rosenfeld and J. Kruger, J. Appl. Phys., 2009, 106, 104910-104915.

57. D. Ashkenasi, A. Rosenfeld, H. Varel, M. Wähmer and E. E. B. Campbell, Appl. Surf. Sci., 1997, 120, 65-80.

15 58. L. V. Keldysh, Sov. Phys. JETP, 1965, 20, 1307.

59. N. Tsutsumi and A. Fujihara, J. Appl. Phys., 2007, 101, 033110033115 . 